Homology, Homotopy and Applications, vol.10(1), 2008, pp.223-236

\title{
GROUP ELEMENTS CONJUGATE TO THEIR POWERS AND BASS' CONJECTURE
}

\author{
DIMITRIOS PANAGOPOULOS
}

(communicated by Jean-Louis Loday)

\begin{abstract}
In this paper, we use some specific properties that are enjoyed by group elements with non-zero Hattori-Stallings rank function and define, by homological means, a large class of groups that satisfy Bass' conjecture. We examine closure properties of that class and exhibit certain examples of groups which are contained therein.
\end{abstract}

\section{Introduction}

In 1976 Hyman Bass [1, 2] formulated a conjecture which can be expressed as follows:

Let $k$ be a subring of $\mathbb{C}$ with $k \cap \mathbb{Q}=\mathbb{Z}$ and let $G$ be a group. Then, for every element $g \in G$ with $g \neq 1$, the Hattori-Stallings rank function $r_{g}: K_{0}(k G) \rightarrow k$ is identically zero.

Linnell [13], Schafer [18] and Moody [16] proved that if $r_{g}$ is not identically zero then the element $g$ has some very specific properties; in particular, it turns out that $r_{g}=0$ if $g \neq 1$ and $g$ has finite order. Using results of Karoubi [12], Connes [5] and Burghelea [4], it follows that if $g$ has infinite order, then the map $r_{g}$ can be factored through the homology group $H_{2 n}(N(g), k)$, where $N(g)=C(g) /\langle g\rangle$ and $C(g)$ is the centralizer of $g$ in $G$. More precisely, there is a commutative diagram

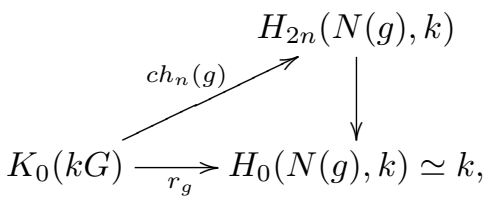

where $c h_{n}(g)$ is a component of the so-called Connes-Karoubi character map

$$
c h_{n}: K_{0}(k G) \rightarrow H C_{2 n}(k G)
$$

and $H C_{2 n}(k G)$ is the $2 n$-th cyclic homology group of the group algebra $k G$. Here, the map

Received June 3, 2007, revised January 9, 2008; published on February 25, 2008.

2000 Mathematics Subject Classification: 20J05, 20C07, 16E40.

Key words and phrases: Bass' conjecture, cup product, group acting on trees.

This article is available at http://intlpress.com/HHA/v10/n1/a9

Copyright (C) 2008, International Press. Permission to copy for private use granted. 


$$
H_{2 n}(N(g), k) \rightarrow H_{0}(N(g), k) \simeq k
$$

is the cap product map $\alpha(g)^{n} \cap_{-}$, which is induced by the cohomology class $\alpha(g) \in$ $H^{2}(N(g), \mathbb{Z})$ that classifies the central extension

$$
1 \rightarrow \mathbb{Z} \stackrel{g}{\rightarrow} C(g) \rightarrow N(g) \rightarrow 1 .
$$

Using these facts, Eckmann defined in [6] a class of groups satisfying Bass' conjecture, by examining the vanishing of the homology groups $H_{2 n}(N(g), \mathbb{Q})$ for $n \gg 0$. This line of attack was pushed forward by Emmanouil and Passi [7, 10]. Motivated by the class $\mathcal{A}$ defined in $[\mathbf{1 0}]$, we define in this paper a new class $\mathcal{A}_{L}$ of groups that satisfy Bass' conjecture. In order to examine whether a given group belongs to $\mathcal{A}_{L}$, we restrict our attention only to those group elements that have Linnell's property. This allows us to prove, in a relatively simple way, that certain groups satisfy Bass' conjecture by showing that they are contained in $\mathcal{A}_{L}$.

Following its definition, in Section 2 we examine closure properties of the new class. In particular, it turns out that $\mathcal{A}_{L}$ is residually closed. In Section 3 we mention some group theoretic facts concerning elements in amalgamated free products and HNNextensions that have Linnell's property. These facts will be used in the following section in order to obtain some concrete examples of groups in $\mathcal{A}_{L}$ (which thereby satisfy Bass' conjecture). Furthermore, in Section 4, we show that every countable group in $\mathcal{A}_{L}$ can be embedded in a group with two generators that is also contained in $\mathcal{A}_{L}$.

\section{Notation}

Given an element $g$ of a group $G$, we denote by $C_{G}(g)$ (or $C(g)$ when the group $G$ is easily understood) the centralizer of $g$ in $G$. We also denote by $N_{G}(g)$ (or $N(g)$ ) the quotient $C_{G}(g) /\langle g\rangle$ and let $\alpha(g)$ be the element in $H^{2}(N(g), \mathbb{Z})$ that classifies the central extension

$$
1 \longrightarrow \mathbb{Z} \stackrel{g}{\longrightarrow} C_{G}(g) \longrightarrow N_{G}(g) \longrightarrow 1
$$

\section{Acknowledgements}

The results of this paper were obtained as part of the author's Ph.D. thesis at the University of Athens. I would like to thank my advisor I. Emmanouil as well as D. Varsos for their helpful comments and suggestions. I would also like to thank the anonymous referee for pointing out that Bass-Serre theory could simplify and extend some of the group theoretic results of this paper.

\section{Definition of $\mathcal{A}_{L}$ and basic closure properties}

In this section we introduce the class $\mathcal{A}_{L}$ and study some of its closure properties.

Definition 2.1. Let $G$ be a group. We say that $g \in G$ has Linnell's property if there exists $u \in \mathbb{N}, u \neq 0$, such that the elements $g$ and $g^{n^{u}}$ are conjugate for all $n \in \mathbb{N}$, $n \geqslant 1$.

It is immediate from the definition that the following lemma holds. 
Lemma 2.2. Let $G$ be a group and $g \in G$ an element having Linnell's property. Then:

(i) if $g \neq 1$, then $g$ has infinite order,

(ii) if $\phi: G \rightarrow H$ is a homomorphism, then $\phi(g)$ has Linnell's property,

(iii) if $K \unlhd G$, then either $g \in K$ or else $\langle g\rangle \cap K=\{1\}$,

(iv) for every $n \in \mathbb{N}, n \geqslant 1$, there exists $h \in G$ such that $g=h^{n}$.

\section{Definition 2.3.}

(i) $\mathcal{A}_{L}$ The class $\mathcal{A}_{L}$ consists of those groups $G$ that satisfy the following condition: for every element $g \in G \backslash\{1\}$ having Linnell's property there exists $n \in \mathbb{N}$ such that the cap product map

$$
\alpha(g)^{n} \cap_{-}: H_{2 n}(N(g), \mathbb{Z}) \rightarrow H_{0}(N(g), \mathbb{Z}) \simeq \mathbb{Z}
$$

is not surjective.

(ii) Let $R \mathcal{A}_{L}$ be the class of groups that residually belong to $\mathcal{A}_{L}$.

(iii) Let $\mathcal{A}$ be the class of groups with the property that for every element $g \in G$ of infinite order there exists $n \in \mathbb{N}$ such that the cap product map (1) is not surjective (cf. [10]).

Obviously $\mathcal{A} \subseteq \mathcal{A}_{L}$. Furthermore, since Linnell's property is a rather strong condition for an element of a group, it follows immediately that certain types of groups belong to $\mathcal{A}_{L}$. In particular, a group $G$ is contained in $\mathcal{A}_{L}$ if there is no element $g \in G$, $g \neq 1$, having Linnell's property. For example, finite, abelian, abelian by finite, torsion, residually free groups (cf. $[\mathbf{3}]$ ) and more generally residually finite groups (cf. [9, Corollary 3.38]) belong to $\mathcal{A}_{L}$. We should note here that Sykiotis in [22] and Strojnowski in [20] studied groups whose elements do not have Linnell's property.

The relevance of the cap product map (1) in the study of the Hattori-Stallings rank function $r_{g}: K_{0}(k G) \rightarrow k$ stems from the following result.

Lemma 2.4 (cf. [10, Lemma 4.1]). Let $k$ be a commutative ring, which is torsion-free as an abelian group. We consider a group $G$, an element $g \in G$ of infinite order and assume that there exists a positive integer $n$, such that the cap product map (1) is not surjective. Then, the Hattori-Stallings rank function $r_{g}: K_{0}(k G) \rightarrow k$ is zero.

Working as in [10, Theorem 4.2], we may now obtain the following result.

Theorem 2.5. Groups in $\mathcal{A}_{L}$ satisfy Bass' conjecture.

Proof. Let $G \in \mathcal{A}_{L}$. If there exist a subring $k \subseteq \mathbb{C}$ with $k \cap \mathbb{Q}=\mathbb{Z}$ and a non-trivial element $g \in G$ such that the Hattori-Stallings rank $r_{g}: K_{0}(k G) \rightarrow k$ is not zero, then $g$ has Linnell's property $[\mathbf{1 3}, \mathbf{1 6}, \mathbf{1 8}]$. Since $G \in \mathcal{A}_{L}$, it follows that the cap product map (1) is not surjective for some $n \in \mathbb{N}$. In view of Lemma 2.4, we conclude that the Hattori-Stallings rank function $r_{g}: K_{0}(k G) \rightarrow k$ is zero, contrary to our hypothesis.

Proposition 2.6. The class $\mathcal{A}_{L}$ is closed under:

(i) subgroups,

(ii) free products,

(iii) direct products. 
Proof.

(i) The result follows from [10, Remark $3.2($ ii) $]$ by considering the inclusion map $\iota: H \hookrightarrow G$, since whenever $g \in H$ has Linnell's property (in $H$ ) then $g$ has Linnell's property in $G$.

(ii) The result follows as in [7, Proposition 3.5(ii)] with the additional observation that, due to the normal form theorem for free products, if $g, h$ are conjugates in $G_{1} * G_{2}$ with $g, h$ in one factor, then they are conjugates in the same factor.

(iii) Let $G=\prod_{i} G_{i}$ with $G_{i} \in \mathcal{A}_{L}$ and $g=\left(g_{i}\right) \in G, g \neq 1$, be an element having Linnell's property. Applying Lemma 2.2(ii) to the projection $\pi_{i}: G \rightarrow G_{i}$, we conclude that the element $g_{i} \in G_{i}$ has Linnell's property for every $i$. Since $g \neq 1$, there must exist an $i$ such that $g_{i} \neq 1_{G_{i}}$. For that particular $i$ there exists $n \in \mathbb{N}$ such that the cap product map

$$
\alpha\left(g_{i}\right)^{n} \cap_{-}: H_{2 n}\left(N_{G_{i}}\left(g_{i}\right), \mathbb{Z}\right) \rightarrow H_{0}\left(N_{G_{i}}\left(g_{i}\right), \mathbb{Z}\right) \simeq \mathbb{Z}
$$

is not surjective. It follows that the cap product map (1) is not surjective either (cf. [10, Remark 3.2(ii)]). Hence, we conclude that $G \in \mathcal{A}_{L}$.

Remark 2.7. The main argument used in the proof of assertion (ii) above, which is proved in [7, Appendix A], concerns the centralizer of an element in a free product, which is not conjugate to an element in a factor. As we will see later (Corollary 3.4), the proof of assertion $(i i)$ can be generalized to certain amalgamated free products.

Corollary 2.8. $\mathcal{A}_{L}=R \mathcal{A}_{L}$.

Proof. It is obvious that $\mathcal{A}_{L} \subseteq R \mathcal{A}_{L}$. On the other hand, every class of groups which is closed under subgroups and direct products is residually closed. Indeed, let $G$ be a group that is residually contained in $\mathcal{A}_{L}$. Then, for every element $g \in G \backslash\{1\}$ there exists a group $H_{g}$ which belongs to $\mathcal{A}_{L}$ and a homomorphism $\phi_{g}: G \rightarrow H_{g}$, such that $\phi_{g}(g) \neq 1$. We therefore have the embedding $\phi: G \hookrightarrow \prod_{g \neq 1} H_{g}$ with $\phi(h)=\left(\phi_{g}(h)\right)_{g}$. Thus, $G$ belongs to $\mathcal{A}_{L}$ since it can be viewed as a subgroup of $\prod_{g \neq 1} H_{g}$.

Working along the same lines as in [8, Lemma 3.4 and Corollary 3.5], we have the following two results that concern the closure of $\mathcal{A}_{L}$ under extensions.

Proposition 2.9. An extension of a group in $\mathcal{A}_{L}$ by a torsion group is also contained in $\mathcal{A}_{L}$.

Proof. Indeed, let $H$ be a torsion normal subgroup of a group $G$ such that $\bar{G}=$ $G / H \in \mathcal{A}_{L}$. If $g \in G, g \neq 1$, has Linnell's property, then $g \notin H$ and hence $g H \in \bar{G}$ is a non-identity element having Linnell's property; in particular, $g H$ has infinite order (cf. Lemma 2.2). The projection $G \rightarrow \bar{G}$ induces the following commutative diagram of central extensions

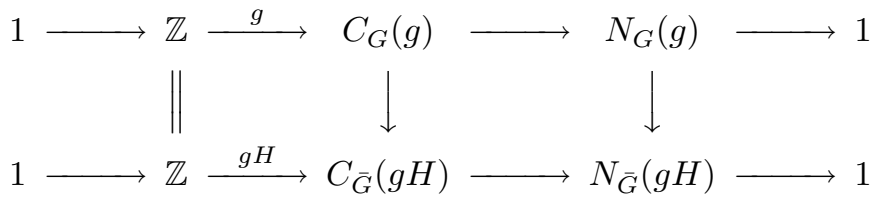

and hence the element $\alpha(g) \in H^{2}\left(N_{G}(g), \mathbb{Z}\right)$ that classifies the top row is a pullback of the element $\alpha(g H) \in H^{2}\left(N_{\bar{G}}(g H), \mathbb{Z}\right)$ that classifies the bottom row. Since $\bar{G} \in \mathcal{A}_{L}$, 
the cap product map $\alpha(g H)^{n} \cap$ _ is not surjective for some $n \in \mathbb{N}$. The same is true for $\alpha(g)^{n} \cap_{-}$, in view of $[\mathbf{1 0}$, Remark 3.2(ii)].

Proposition 2.10. A central extension of a group in $\mathcal{A}_{L}$ is also contained in $\mathcal{A}_{L}$.

Proof. Let $H$ be a central subgroup of a group $G$, such that $G / H \in \mathcal{A}_{L}$, and consider an element $g \in G \backslash\{1\}$ having Linnell's property. Since $H$ is central in $G, g$ is not contained in $H$. Therefore, Lemma 2.2( $i i)$ implies that $g H$ is a non-identity element of $G / H$ having Linnell's property. Since $G / H \in \mathcal{A}_{L}$ we may use the same argument as in the proof Proposition 2.9, in order to conclude that the cap product map $\alpha(g)^{n} \cap$ is not surjective for some $n \in \mathbb{N}$.

\section{Elements having Linnell's property in amalgams and HNN- extensions}

In this section we will use some group theoretic results on generalized free products of groups, in order to provide some examples of groups in $\mathcal{A}_{L}$.

We recall that according to the Bass-Serre theory of groups acting on trees $[\mathbf{1 9}$, I.5.4], for every amalgam of groups $G=A *_{H} B$ (or HNN-extension $G=\langle A, t|$ $\left.\left.t^{-1} H t=H^{\prime}\right\rangle\right)$, there exists a tree $T$ on which the group $G$ acts without inversions, such that the vertex stabilizers are conjugates of $A$ or $B$ (or just $A$ in the case of an HNN-extension) and the edge stabilizers are conjugates of $H$. The distance $d(x, y)$ between two vertices $x, y$ of $T$ is the length of the unique path (also called geodesic) connecting them. For an element $g$ in $G$ the translation length is the number $\tau(g)=\min \{d(x, g x) \mid x \in T\}$. The set $T_{g}=\{x \in T \mid d(x, g x)=\tau(g)\}$ constitutes the vertex set of a subtree of $T$. An element $g$ in $G$ is called elliptic if $\tau(g)=0$ and in that case $T_{g}$ is the set of fixed points of $g$. If $\tau(g)>0$, then the element $g$ is called hyperbolic and in that case $T_{g}$ is a line on which $g$ acts with amplitude $\tau(g)[\mathbf{1 9}$, I.6.4, Prop. 24]. Since every element of $G$ can be seen as an isometry of $T$ via the group action, it follows that the translation lengths of two conjugate elements are equal. Furthermore, $\tau\left(g^{n}\right)=n \tau(g)$ for every $g \in G$ and $n \in \mathbb{N}$.

Lemma 3.1. Let $G=A *_{H} B$ be an amalgam (or $G=\left\langle A, t \mid t^{-1} H t=H^{\prime}\right\rangle$ an $H N N$ extension) and $g \in G \backslash\{1\}$ an element having Linnell's property. Then $g$ is conjugate to an element of $A$ or $B$ (or just $A$ in the case of an HNN-extension). Furthermore, if $g$ is not conjugate to an element of $H$, then it has Linnell's property in that conjugate of $A$ or $B$ (or just $A$ in the case of an HNN-extension).

Proof. Let $T$ be the Bass-Serre tree associated to the group $G$. Since $g$ has Linnell's property, there exists a positive natural number $u$ such that $g$ and $g^{n^{u}}$ are conjugate for all $n \geqslant 1$. Then $\tau(g)=\tau\left(g^{n^{u}}\right)=n^{u} \tau(g)$ and hence $\tau(g)=0$. Therefore, $g$ is elliptic and fixes a vertex $x$. Since the stabilizers of vertices are conjugates of $A$ or $B$ it follows that $g$ is contained in a conjugate of $A$ or $B$ (or just $A$ in the case of an HNN-extension).

If $g$ is not conjugate to an element in $H$ it cannot fix two distinct vertices $x, y$, for then it would fix the geodesic from $x$ to $y$. It would then had to stabilize an edge and it would follow that $g$ is in a conjugate of $H$. Now for a fixed $n$ let $h \in G$ be an element such that $g=h g^{n^{u}} h^{-1}$. Then the vertex $h x$ is stabilized by $g$ since 
$g h x=h g^{n^{u}} h^{-1} h x=h g^{n^{u}} x=h x$. Thus $h x=x$ and it follows that $h$ is contained in the stabilizer $G_{x}$ of $x$. Hence, $g$ has Linnell's property in $G_{x}$.

Remark 3.2. Let $G$ be a group acting on a tree $T$ as in the proof of the previous lemma. For every element $g \in G$ we may observe that the centralizer $C_{G}(g)$ of $g$ acts on $T_{g}$. That is because $d(h x, g h x)=d(h x, h g x)=d(x, g x)$ for every $h \in C_{G}(g)$, since $h$ acts as an isometry on $T$. In particular, if $T_{g}=\{x\}$ then it follows that $C_{G}(g)$ fixes $x$ and hence it is a subgroup of $G_{x}$, i.e. $C_{G}(g)$ is in the same conjugate of $A$ or $B$ (or just $A$ ) as the element $g$.

Remark 3.3. Let $G=A *_{H} B$ be the amalgam of two groups $A, B \in \mathcal{A}_{L}$ along a common subgroup $H$ of theirs (or $G=\left\langle A, t \mid t^{-1} H t=H^{\prime}\right\rangle$ an HNN-extension of a group $\left.A \in \mathcal{A}_{L}\right)$. In order to show that $G \in \mathcal{A}_{L}$, we have to examine elements $g \in G$ having Linnell's property. In view of Lemma 3.1 and Remark 3.2, we have to consider the following two cases:

(i) The element $g$ is conjugate to an element $g^{\prime}$, which is contained in a factor but is not conjugate to an element of $H$. In this case, the centralizer of $g$ is conjugate to the centralizer of $g^{\prime}$, which is contained in that factor. Furthermore, $g^{\prime}$ has Linnell's property and so the cap product map (1) is not surjective for some $n \gg 0$ ( since the factor is contained in $\mathcal{A}_{L}$ ).

(ii) The element $g$ is conjugate to an element $g^{\prime} \in H$. It is in this case that it may happen that the cap product map (1) is surjective for all $n \in \mathbb{N}$.

In particular, we obtain the following corollary which generalizes Proposition 2.6(ii).

Corollary 3.4. If $A, B \in \mathcal{A}_{L}$ and $H$ is a common torsion subgroup of theirs, then $A *_{H} B \in \mathcal{A}_{L}$. Moreover, if $H^{\prime} \subseteq A$ is a subgroup isomorphic with $H$, then

$$
\left\langle A, t \mid t^{-1} H t=H^{\prime}\right\rangle \in \mathcal{A}_{L} .
$$

We note that Sykiotis has proved in [21, Theorem 3.1] the statement corresponding to Corollary 3.4 for the class of groups defined in [7] instead of $\mathcal{A}_{L}$.

For use in the following section, we note the following.

Definition 3.5. Let $G *=\left\langle G, t \mid t^{-1} A t=B, \phi\right\rangle$ be an HNN-extension. Pinch is a word of the form $t^{-\varepsilon} u t^{\varepsilon}$, where $u \in A$ if $\varepsilon=1$ and $u \in B$ if $\varepsilon=-1$. An element $g=g_{0} t^{\varepsilon_{1}} g_{1} \ldots t^{\varepsilon_{n}} g_{n} \in G *$ that does not contain a pinch is called reduced. In that case the number $|g|=n$ is called the length of $g$.

Lemma 3.6. Let $G *=\left\langle G, t \mid t^{-1} A t=B, \phi\right\rangle$ be an $H N N$-extension and $u \in G \subseteq G *$. If $C_{G *}(u)$ contains an element $g$ with $g \notin G$, then $u$ is conjugate with an element in $A$ (and thus it is conjugate with an element in $B$ as well).

Proof. Let $g=g_{0} t^{\varepsilon_{1}} g_{1} \ldots t^{\varepsilon_{n}} g_{n} \in C_{G *}(u)$ in normal form. Then,

$$
u^{-1} g_{n}^{-1} t^{-\varepsilon_{n}} \ldots t^{-\varepsilon_{1}} g_{0}^{-1} u g_{0} t^{\varepsilon_{1}} g_{1} \ldots t^{\varepsilon_{n}} g_{n}=1(*)
$$

and hence there must exist a pinch. Since $g$ is in normal form, it is only $t^{-\varepsilon_{1}} g_{0}^{-1} u g_{0} t^{\varepsilon_{1}}$ that can be a pinch. Hence, $z_{1}=t^{-\varepsilon_{1}} g_{0}^{-1} u g_{0} t^{\varepsilon_{1}} \in A$ or $B$. Then, (*) becomes

$$
u^{-1} g_{n}^{-1} t^{-\varepsilon_{n}} \ldots t^{-\varepsilon_{2}} g_{1}^{-1} z_{1} g_{1} t^{\varepsilon_{2}} g_{2} \ldots t^{\varepsilon_{n}} g_{n}=1
$$

and continuing like this we arrive at $u^{-1} g_{n}^{-1} z_{n} g_{n}=1$ with $z_{n} \in A$ or $B$. Therefore, $u$ is conjugate with an element in $A$ or $B$. 
We should note here that results similar to those in this section can be obtained using [15, Chapter 4, Theorems 4.5 and 4.6] and [14, Chapter IV, Theorem 2.5], by noticing that the length of powers of elements grows.

\section{Two examples of groups in $\mathcal{A}_{L}$}

\subsection{An amalgam in $\mathcal{A}_{L}$}

In this subsection we will show that the group $K$ constructed in [8, pages 168-170] is contained in $\mathcal{A}_{L}$. In particular, it will follow that $K$ satisfies Bass' conjecture. First of all, we give a brief description of its construction.

Let $\Gamma=\mathbb{Z} \imath \mathbb{Z}$ be the wreath product of $\mathbb{Z}$ by $\mathbb{Z}$. We have the following short exact sequence:

$$
1 \rightarrow N \rightarrow \Gamma \rightarrow \mathbb{Z} \rightarrow 1
$$

where $N=\bigoplus_{i=-\infty}^{+\infty} \mathbb{Z} e_{i}$ and $\mathbb{Z} \simeq\langle\psi\rangle$ acts on $N$ in such a way that $\psi\left(e_{i}\right)=e_{i+1}$ for all $i \in \mathbb{Z}$. In view of [10, Proposition 5.3], the group $\Gamma$ is contained in $\mathcal{A} \subseteq \mathcal{A}_{L}$. In [8, Proposition 2.1] and [11, Paragraph 3] it is proved that if $k$ is a commutative ring, then $H^{n}(N, k)$ is the inverse $\operatorname{limit} \lim _{\leftarrow t} \Lambda_{t}^{n}$, where $\Lambda_{t}^{n}$ is the component of degree $n$ of the $k$-exterior algebra generated by degree 1 elements $\left\{f_{i}\right\}_{i=-t}^{t}$.

If $\beta=\sum_{i=-\infty}^{+\infty} f_{i} \wedge f_{i+1} \in H^{2}(N, \mathbb{Z})$, then $\beta^{n} \in H^{2 n}(N, \mathbb{Z})$ is the formal sum consisting of terms of the form $n ! f_{i_{1}} \wedge f_{i_{1}+1} \wedge \ldots \wedge f_{i_{n}} \wedge f_{i_{n}+1}$ with $i_{1}+1<i_{2}, \ldots, i_{n-1}$ $+1<i_{n}$; hence, $\beta$ is not nilpotent. Furthermore, as discussed in [11, Paragraph 3], $\beta \in \operatorname{Im}\left(H^{2}(\Gamma, \mathbb{Z}) \stackrel{\text { res }}{\rightarrow} H^{2}(N, \mathbb{Z})\right)$ and hence we can view $\beta$ as an element in $H^{2}(\Gamma, \mathbb{Z})$.

Let $\alpha=\iota_{*}(\beta)$, where $\iota: \mathbb{Z} \rightarrow \mathbb{Q}$ is the inclusion, classify the central extension

$$
1 \rightarrow \mathbb{Q} \stackrel{1 \mapsto g}{\longrightarrow} G \rightarrow \Gamma \rightarrow 1 .
$$

Then, Proposition 2.10 implies that $G \in \mathcal{A}_{L}$. In fact, we shall prove the following.

Proposition 4.1. The group $G$ is contained in $\mathcal{A}$.

Proof. Let $x \in G$ be an element of infinite order. We will show that the cap product map (1) is not surjective for some $n \in \mathbb{N}$. The proof splits in two cases.

$x \notin \mathbb{Q}$ : Since $\Gamma$ is torsion-free, we conclude that if we consider the projection $\pi: G \rightarrow$ $\Gamma$ the element $\pi(x)$ is of infinite order and we have the following commutative diagram of central extensions:

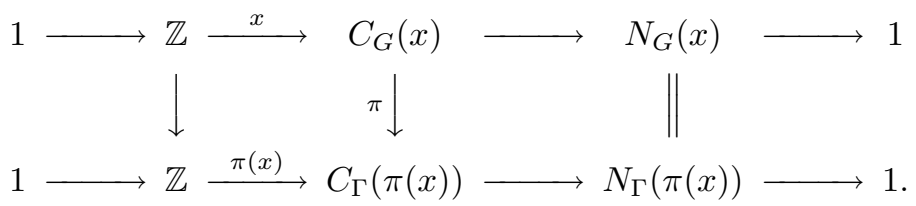

Since $\Gamma \in \mathcal{A}$, the cap product map $\alpha[\pi(x)]^{n} \cap$ _ associated with the bottom row 
is not surjective for $n \gg 0$. In view of [10, Remark 3.2(ii)], it follows that this is also true for the cap product map $\alpha(x)^{n} \cap_{-}$.

$x \in \mathbb{Q}$ : Then $x=r g, r \in \mathbb{Q}, r \neq 0$, and we obtain the following commutative diagram of central extensions:

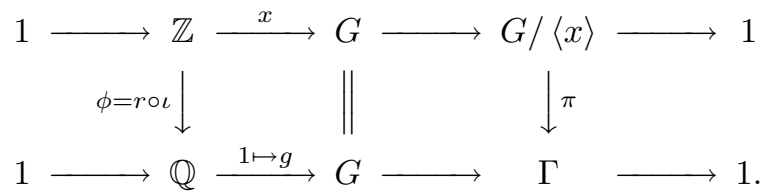

Here $\phi: \mathbb{Z} \rightarrow \mathbb{Q}$ is the additive map with $\phi(1)=r$ and $\iota: \mathbb{Z} \rightarrow \mathbb{Q}$ the inclusion. Let $\gamma=\alpha(x)$ classify the extension

$$
1 \longrightarrow \mathbb{Z} \stackrel{x}{\longrightarrow} G \longrightarrow G /\langle x\rangle \longrightarrow 1 \text {. }
$$

Then, $\pi^{*}(\alpha)=r \iota_{*}(\gamma)$ and hence we have $\pi^{*}(\alpha)^{n}=r^{n} \iota_{*}(\gamma)^{n}$ for all $n \geqslant 0$. In particular, there is a commutative diagram:

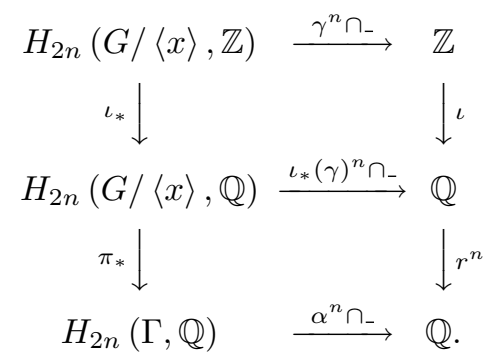

If the cap product map at the top $\gamma^{n} \cap_{-}: H_{2 n}(G /\langle x\rangle, \mathbb{Z}) \rightarrow \mathbb{Z}$ is surjective for all $n \in \mathbb{N}$, then $r^{n} \in \operatorname{Im}\left(\left(\alpha^{n} \cap_{-}\right) \circ \pi_{*} \circ \iota_{*}\right)$ for all $n \in \mathbb{N}$.

Since $\alpha=\iota_{*}(\beta)$, we have the following commutative diagram:

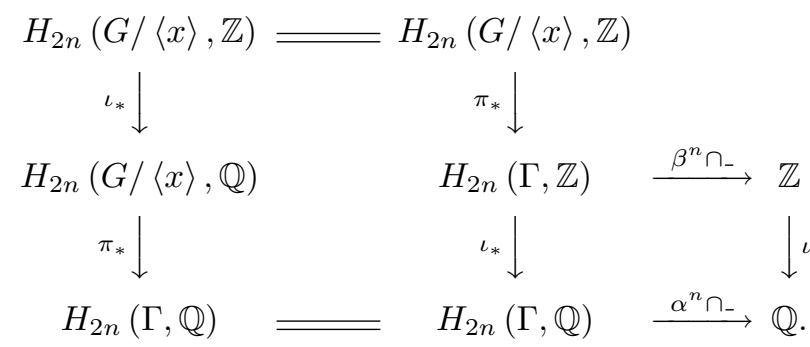

Furthermore (cf. [11, Paragraph 3]), $\beta^{n}=n ! \sum f_{i_{1}} \wedge f_{i_{1}+1} \wedge \ldots \wedge f_{i_{n}} \wedge f_{i_{n}+1}$, where the sum runs over the sequences $\left(i_{1}, \ldots, i_{n}\right)$ with $i_{1}+1<i_{2}, \ldots, i_{n-1}+$ $1<i_{n}$. Since the element $\sum f_{i_{1}} \wedge f_{i_{1}+1} \wedge \ldots \wedge f_{i_{n}} \wedge f_{i_{n}+1} \in H^{2 n}(N, \mathbb{Z})$ is contained in the image of res: $H^{2 n}(\Gamma, \mathbb{Z}) \rightarrow H^{2 n}(N, \mathbb{Z})$, it follows that $\operatorname{Im}\left(\beta^{n} \cap_{-}\right) \subseteq$ $n ! \mathbb{Z}$.

Since $\left(\alpha^{n} \cap_{-}\right) \circ \pi_{*} \circ \iota_{*}=\left(\alpha^{n} \cap_{-}\right) \circ \iota_{*} \circ \pi_{*}=\iota \circ\left(\beta^{n} \cap_{-}\right) \circ \pi_{*}$, we conclude that $\operatorname{Im}\left(\left(\alpha^{n} \cap_{-}\right) \circ \pi_{*} \circ \iota_{*}\right)=\operatorname{Im}\left(\iota \circ\left(\beta^{n} \cap_{-}\right) \circ \pi_{*}\right) \subseteq n ! \mathbb{Z}$.

But $r^{n} \in \operatorname{Im}\left(\left(\alpha^{n} \cap_{-}\right) \circ \pi_{*} \circ \iota_{*}\right)$ and hence $r^{n} \in n ! \mathbb{Z}$ for all $n \in \mathbb{N}$. Equivalently, we have $\frac{r^{n}}{n !} \in \mathbb{Z}$ for all $n \in \mathbb{N}$, which is absurd since $\frac{r^{n}}{n !}$ tends to zero as $n$ tends 
to infinity. This completes the proof of the assertion that the cap product map $\gamma^{n} \cap_{-}: H_{2 n}(G /\langle x\rangle, \mathbb{Z}) \rightarrow \mathbb{Z}$ is not surjective for $n \gg 0$ in this case as well.

Let $G^{\prime}=G \times\left(\bigoplus_{i \in \mathbb{Z}^{*}} \mathbb{Q} u_{i}\right)$ and consider the following commutative diagram of central extensions:

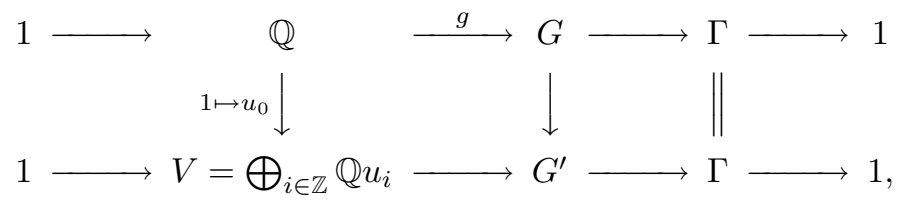

as in $[8]$. We have the following result.

Corollary 4.2. The group $G^{\prime}$ is contained in $\mathcal{A}$.

Proof. This is immediate from the fact that $G$ as well as the abelian group $\bigoplus_{i \in \mathbb{Z}^{*}} \mathbb{Q} u_{i}$ are both contained in $\mathcal{A}$, which is itself closed under finite direct products (cf. [10, Proposition 5.1]).

Let $\mathcal{H}$ be Hall's group. Recall that $\mathcal{H}$ is the semidirect product of $V=\bigoplus_{i \in \mathbb{Z}} \mathbb{Q} u_{i}$ and $\Lambda=\langle x, y\rangle \subseteq \operatorname{Aut}_{\mathbb{Q}}(V)$, where $x\left(u_{i}\right)=u_{i+1}$ and $y\left(u_{i}\right)=p_{i} u_{i}$ for all $i \in \mathbb{Z}$; here, $\left\{p_{i}: i \in \mathbb{Z}\right\}$ is an enumeration of all primes. In view of $[\mathbf{8}$, Lemma 4.2$]$, we have $\mathcal{H} \in \mathcal{A}$.

Let $K=G^{\prime} *_{V} \mathcal{H}$. In the very end of [8] it was stated that "one cannot prove whether $K$ satisfies Bass' conjecture using either Linnell's result or the cyclic homology approach". We will prove that $K \in \mathcal{A}_{L}$; in particular, it will follow that $K$ does satisfy Bass' conjecture. To that end, we will need the following lemmas.

Lemma 4.3. Let $0 \neq u \in V \leqslant K$. If $g \in C_{K}(u)$ and its normal form is

$$
\phi_{1} g_{1} \ldots \phi_{n} g_{n} h
$$

where $g_{i} \in G^{\prime}$ are coset representatives of $V$ in $G^{\prime}, \phi_{i} \in \Lambda$ are coset representatives of $V$ in $\mathcal{H}$ and $h \in V$ (for notational purposes we use this slightly changed "normal" form, where it is possible to have $\phi_{1}=1$ or $\left.g_{n}=1\right)$, then $\phi_{1} \ldots \phi_{n}=1_{\Lambda}$. Conversely, any element of $K$ with normal form $\phi_{1} g_{1} \ldots \phi_{n} g_{n} h$, such that $\phi_{1} \ldots \phi_{n}=1_{\Lambda}$, is contained in the centralizer $C_{K}(u)$ of $u$.

Proof. We note that the following assertions are valid for all elements $v \in V$ :

(i) $g v=v g$ for all $g \in G^{\prime}$, since $V$ is central in $G^{\prime}$,

(ii) $v \phi_{i}=\phi_{i} \phi_{i}^{-1}(v)$ for all $\phi_{i} \in \Lambda$, since

$$
\phi_{i}{ }^{-1} v \phi_{i}=\left(0, \phi_{i}{ }^{-1}\right)\left(v, 1_{\Lambda}\right)\left(0, \phi_{i}\right)=\left(\phi_{i}^{-1}(v), 1_{\Lambda}\right)=: \phi_{i}^{-1}(v) .
$$

Let $\phi_{1} g_{1} \ldots \phi_{n} g_{n} h \in C_{K}(u)$. Then, by using $(i)$ and $(i i)$ above, we obtain that

$$
\phi_{1} g_{1} \ldots \phi_{n} g_{n} h u h^{-1} g_{n}^{-1} \phi_{n}^{-1} \ldots g_{1}^{-1} \phi_{1}^{-1}=\phi_{1} \ldots \phi_{n}(u)
$$

and hence $\phi_{1} \ldots \phi_{n}(u)=u \in K$. In view of the normal form theorem for amalgams, we have $\phi_{1} \ldots \phi_{n}(u)=u \in \mathcal{H}$ and finally, since the stabilizer of $u$ in $\mathcal{H}$ is equal to $\left\{1_{\Lambda}\right\}$ (cf. [9, page 104]), we conclude that $\phi_{1} \ldots \phi_{n}=1 \in \mathcal{H}$.

Conversely, let $\phi_{1} g_{1} \ldots \phi_{n} g_{n} h$ be an element of $K$ with $\phi_{1} \ldots \phi_{n}=1 \in \Lambda$. Then, by using $(i)$ and $(i i)$ we have $u \phi_{1} g_{1} \ldots \phi_{n} g_{n} h=\phi_{1} g_{1} \ldots \phi_{n} g_{n} h u$. 
Corollary 4.4. For any $u \in V \backslash\{0\}$, the centralizer $C_{K}(u)$ is the normal subgroup of $K$ generated by the elements of $G^{\prime}$.

Lemma 4.5. Let $u \in V, u \neq 0$. Then, the map $\pi: C_{K}(u) \rightarrow G^{\prime}$ which sends any element $\phi_{1} g_{1} \ldots \phi_{n} g_{n} h \in C_{K}(u)$ to $g_{1} g_{2} \ldots g_{n} h$ is a surjective group homomorphism.

Proof. The map is well-defined since from the normal form theorem every $x \in K$ can be written in a unique way as $\phi_{1} g_{1} \ldots \phi_{n} g_{n} h$.

Let $x=\phi_{1} g_{1} \ldots \phi_{n} g_{n} h, x^{\prime}=\phi_{1}^{\prime} g_{1}^{\prime} \ldots \phi_{m}^{\prime} g_{m}^{\prime} h^{\prime} \in C_{K}(u)$. Then, $\pi(x)=g_{1} \ldots g_{n} h$ and $\pi\left(x^{\prime}\right)=g_{1}^{\prime} \ldots g_{m}^{\prime} h^{\prime}$. It follows that

$$
\pi(x) \pi\left(x^{\prime}\right)=g_{1} \ldots g_{n} h g_{1}^{\prime} \ldots g_{m}^{\prime} h^{\prime}=g_{1} \ldots g_{n} g_{1}^{\prime} \ldots g_{m}^{\prime} h h^{\prime}
$$

Since $\phi_{1}^{\prime} \ldots \phi_{m}^{\prime}=1$, the latter product equals

$$
\pi\left(\phi_{1} g_{1} \ldots \phi_{n} g_{n} \phi_{1}^{\prime} g_{1}^{\prime} \ldots \phi_{m}^{\prime} g_{m}^{\prime} h h^{\prime}\right)=\pi\left(\phi_{1} g_{1} \ldots \phi_{n} g_{n} h \phi_{1}^{\prime} g_{1}^{\prime} \ldots \phi_{m}^{\prime} g_{m}^{\prime} h^{\prime}\right)=\pi\left(x x^{\prime}\right)
$$

if neither $g_{n}$ nor $\phi_{1}^{\prime}$ is equal to 1 . If $\phi_{1}^{\prime}=1$, then (using the equality $\phi_{2}^{\prime} \ldots \phi_{m}^{\prime}=1$ ) we have

$$
\begin{aligned}
x x^{\prime} & =\phi_{1} g_{1} \ldots \phi_{n} g_{n} h g_{1}^{\prime} \ldots \phi_{m}^{\prime} g_{m}^{\prime} h^{\prime} \\
& =\phi_{1} g_{1} \ldots \phi_{n} g_{n} g_{1}^{\prime} \ldots \phi_{m}^{\prime} g_{m}^{\prime} h h^{\prime} \\
& =\phi_{1} g_{1} \ldots \phi_{n} z v \phi_{2}^{\prime} \ldots \phi_{m}^{\prime} g_{m}^{\prime} h h^{\prime} \\
& =\phi_{1} g_{1} \ldots \phi_{n} z \phi_{2}^{\prime} \ldots \phi_{m}^{\prime} g_{m}^{\prime} v h h^{\prime} .
\end{aligned}
$$

Here $g_{n} g_{1}^{\prime}=z v$ with $v \in V$ and $z \in G^{\prime}$ a coset representative of $V$ in $G^{\prime}$. Hence, $\pi\left(x x^{\prime}\right)=g_{1} \ldots g_{n-1} z g_{2}^{\prime} \ldots g_{m}^{\prime} v h h^{\prime}$, whereas

$$
g_{1} \ldots g_{n} g_{1}^{\prime} \ldots g_{m}^{\prime} h h^{\prime}=g_{1} \ldots g_{n-1} z v g_{2}^{\prime} \ldots g_{m}^{\prime} h h^{\prime}=g_{1} \ldots g_{n-1} z g_{2}^{\prime} \ldots g_{m}^{\prime} v h h^{\prime} .
$$

If $g_{n}=1$, then (using $\phi_{1}^{\prime} \ldots \phi_{m}^{\prime}=1$ ) we obtain

$$
x x^{\prime}=\phi_{1} g_{1} \ldots g_{n-1} \phi_{n} h \phi_{1}^{\prime} g_{1}^{\prime} \ldots \phi_{m} g_{m}^{\prime} h^{\prime}=\phi_{1} g_{1} \ldots g_{n-1} \phi_{n} \phi_{1}^{\prime} g_{1}^{\prime} \ldots \phi_{m} g_{m}^{\prime} h h^{\prime},
$$

so $\pi\left(x x^{\prime}\right)=g_{1} \ldots g_{n-1} g_{1}^{\prime} \ldots g_{m}^{\prime} h h^{\prime}=g_{1} \ldots g_{n} g_{1}^{\prime} \ldots g_{m}^{\prime} h h^{\prime}$. It follows that $\pi(x) \pi\left(x^{\prime}\right)=$ $\pi\left(x x^{\prime}\right)$ and hence $\pi$ is a homomorphism. Finally it is obvious from the definition that $\pi$ is surjective.

We are ready to prove the following theorem.

Theorem 4.6. The group $K$ constructed above is contained in $\mathcal{A}_{L}$.

Proof. If $x \in K$ is an element having Linnell's property, then Lemma 3.1 implies that $x$ is conjugate with an element of $G^{\prime}$ or $\mathcal{H}$. Since the cap product map that we are interested in is not surjective if the cap product map associated with any conjugate of $x$ is not, we can restrict our attention to those elements in $K$ with Linnell's property, which are contained in a factor of $K$. We distinguish three cases.

$x \in \mathcal{H} \backslash V:$ Remark 3.2 implies that $C_{K}(x)=C_{\mathcal{H}}(x)$ and hence the extension

$$
1 \longrightarrow \mathbb{Z} \stackrel{x}{\longrightarrow} C_{K}(x) \longrightarrow N_{K}(x) \longrightarrow 1
$$

coincides with

$$
1 \longrightarrow \mathbb{Z} \stackrel{x}{\longrightarrow} C_{\mathcal{H}}(x) \longrightarrow N_{\mathcal{H}}(x) \longrightarrow 1 \text {. }
$$

Since $\mathcal{H} \in \mathcal{A}$, the corresponding cap product map (1) is not surjective for some $n \in \mathbb{N}$. 
$x \in G^{\prime} \backslash V$ : Remark 3.2 implies that $C_{K}(x)=C_{G^{\prime}}(x)$ and hence the extension

$$
1 \longrightarrow \mathbb{Z} \stackrel{x}{\longrightarrow} C_{K}(x) \longrightarrow N_{K}(x) \longrightarrow 1
$$

coincides with

$$
1 \longrightarrow \mathbb{Z} \stackrel{x}{\longrightarrow} C_{G^{\prime}}(x) \longrightarrow N_{G^{\prime}}(x) \longrightarrow 1 .
$$

Since $G^{\prime} \in \mathcal{A}$ (Corollary 4.2), the corresponding cap product map (1) is not surjective for some $n \in \mathbb{N}$.

$x \in V$ : We have the following commutative diagram of central extensions:

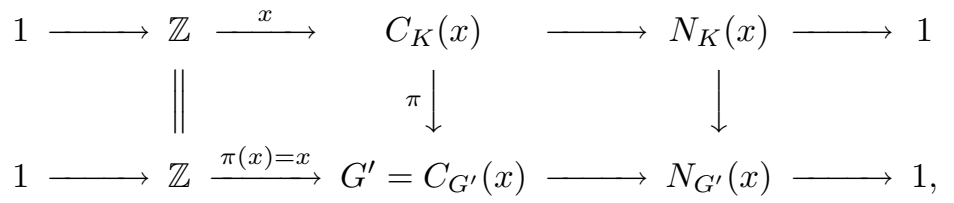

where $\pi: C_{K}(x) \rightarrow G^{\prime}=C_{G^{\prime}}(x)$ is the surjective homomorphism defined in Lemma 4.5. Since $x \in V$, the element $\pi(x)=x$ has infinite order. Let $\beta \in$ $H^{2}\left(N_{G^{\prime}}(x), \mathbb{Z}\right)$ be the element that classifies the bottom row. Since $G^{\prime} \in \mathcal{A}$, the cap product map $\beta^{n} \cap_{-}: H_{2 n}\left(N_{G^{\prime}}(x), \mathbb{Z}\right) \rightarrow H_{0}\left(N_{G^{\prime}}(x), \mathbb{Z}\right) \simeq \mathbb{Z}$ is not surjective for some $n \in \mathbb{N}$. The same is true for the cap product map (1), in view of $[\mathbf{1 0}$, Remark 3.2(ii)].

\subsection{An HNN-extension in $\mathcal{A}_{L}$}

In this subsection we will show that if $G$ is a countably infinite group contained in $\mathcal{A}_{L}$, then $G$ can be embedded in a group $G * \in \mathcal{A}_{L}$, which is generated by two elements. Of course, the latter statement is also true for every finite group, since such a group can be embedded in a permutation group $S_{n}$, for some $n \in \mathbb{N}$.

Theorem 4.7 (Higman, Neumann and Neumann, 1949). Every countable infinite group $G$ can be embedded in a two generator group $G *=\langle t, y\rangle$, where both $t$ and $y$ have infinite order.

Sketch of proof. We slightly modify the argument in the proof of $[\mathbf{1 7}$, Corollary 11.80], by enumerating the infinite group $G$ using the integers instead of the natural numbers. If $G=\left\{g_{i} \mid i \in \mathbb{Z}\right\}$ with $g_{0}=1$ and $F$ is the free group on two generators $x, y$, then

$$
G *=\left\langle G * F, t \mid t^{-1} x^{-n} y x^{n} t=g_{n} y^{-n} x y^{n}, n \in \mathbb{Z}\right\rangle=\left\langle G * F, t \mid t^{-1} A t=B\right\rangle,
$$

where $A=\left\langle x^{-n} y x^{n} \mid n \in \mathbb{Z}\right\rangle$ and $B=\left\langle g_{n} y^{-n} x y^{n} \mid n \in \mathbb{Z}\right\rangle$.

Lemma 4.8. Let $u \in F=\langle x, y\rangle$ be an element such that the sum of the powers of $x$ in $u$ is zero. Then, $u \in A=\left\langle x^{-n} y x^{n} \mid n \in \mathbb{Z}\right\rangle$.

Lemma 4.9. Let $F=\langle x, y\rangle$ be the free group on two generators,

$$
D=\left\langle F, t \mid t^{-1} x^{-n} y x^{n} t=y^{-n} x y^{n}, n \in \mathbb{Z}\right\rangle
$$

and $u, v \in F \subseteq D$ two elements having the following properties:

(i) the sum of the powers of $x$ and the sum of the powers of $y$ in u are equal to zero (i.e. $u \in \ll x \gg \cap \ll y \gg$ ); 
(ii) there exists $f \in D$ with $|f|$ even, such that $f^{-1} u f=v$ (i.e. $u, v$ are conjugates by an element of even length).

Then, there exists $w \in F$ such that $w^{-1} u w=v$ (i.e., $u, v$ are conjugates in $F$ ).

Proof. We note that if an element $w_{1} \in F$ has the property stated in $(i)$ above, then $t^{\varepsilon} w_{1} t^{-\varepsilon}, \varepsilon= \pm 1$ is equal to $\overline{w_{1}} \in F$, where $\overline{w_{1}}$ is the element that we obtain from $w_{1}$ by interchanging $x$ and $y$ in the normal form of $w_{1}$. Furthermore, $\overline{w_{1}}$ also has the property stated in $(i)$ above and $\overline{\overline{w_{1}}}=w_{1}$. Finally, for any two elements $w_{1}, w_{2}$ in $F$ we have $\overline{w_{1} w_{2}}=\overline{w_{1}} \overline{w_{2}}$.

If $f=f_{0} t^{\varepsilon_{1}} f_{1} \cdots t^{\varepsilon_{2 n}} f_{2 n}$ is the normal form of $f$ and $f^{-1} u f=v$, then we have:

$$
f_{2 n}^{-1} t^{-\varepsilon_{2 n}} \cdots t^{-\varepsilon_{1}} f_{0}^{-1} u f_{0} t^{\varepsilon_{1}} \cdots t^{\varepsilon_{2 n}} f_{2 n}=v .
$$

Since $f_{0}^{-1} u f_{0}$ has also the property stated in $(i)$ above, we obtain:

$$
f_{2 n}^{-1} t^{-\varepsilon_{2 n}} \cdots t^{-\varepsilon_{2}} f_{1}^{-1}{\overline{f_{0}}}^{-1} \bar{u} \overline{f_{0}} f_{1} t^{\varepsilon_{2}} \cdots t^{\varepsilon_{2 n}} f_{2 n}=v .
$$

Now $f_{1}^{-1}{\overline{f_{0}}}^{-1} \bar{u} \overline{f_{0}} f_{1}$ has also the same property and hence:

$$
f_{2 n}^{-1} t^{-\varepsilon_{2 n}} \cdots t^{-\varepsilon_{3}} f_{2}^{-1}{\overline{f_{1}}}^{-1} \overline{\overline{f_{0}}} \overline{-1} \overline{\bar{u}} \overline{\overline{f_{0}}} \overline{f_{1}} f_{2} t^{\varepsilon_{3}} \cdots t^{\varepsilon_{2 n}} f_{2 n}=v,
$$

i.e.

$$
f_{2 n}^{-1} t^{-\varepsilon_{2 n}} \cdots t^{-\varepsilon_{3}} f_{2}^{-1}{\overline{f_{1}}}^{-1} f_{0}^{-1} u f_{0} \overline{f_{1}} f_{2} t^{\varepsilon_{3}} \cdots t^{\varepsilon_{2 n}} f_{2 n}=v .
$$

Continuing like this, we arrive to the following equality

$$
f_{2 n}^{-1}{\overline{f_{2 n-1}}}^{-1} \ldots{\overline{f_{1}}}^{-1} f_{0}^{-1} u f_{0} \overline{f_{1}} \cdots \overline{f_{2 n-1}} f_{2 n}=v
$$

which shows that $u, v$ are conjugate in $F$.

Corollary 4.10. Let $G *$ be as in the proof of Theorem 4.7 and $v \neq 1$ an element in $G *$ having Linnell's property in $G *$. Then, $v$ is conjugate to an element $u$ with $C_{G *}(u) \subseteq G * F$. Furthermore, $u$ has Linnell's property in $G * F$.

Proof. By Lemma 3.1, $v$ is conjugate to an element $u \in G * F$. If $C_{G *}(u) \nsubseteq G * F$, then (in view of Lemma 3.6) $u$ is conjugate to an element $w \in A$ that has Linnell's property.

Since $w \in A$, the sum of the powers of $x$ in $w$ is zero. This is also true for the sum of the powers of $y$. Indeed, let $\pi_{1}$ be the projection $\pi_{1}: G * \rightarrow\langle x\rangle$ where $t \mapsto 1$, $h \mapsto 1$ for all $h \in G$ and $x, y \mapsto x$. If the image of $w, \pi_{1}(w)$, is non-trivial, then it is an element of $\langle x\rangle$ other than 1 that has Linnell's property. The last assertion is absurd since $\langle x\rangle$ is the free abelian group in one generator. Therefore, $\pi_{1}(w)=1$ and hence the sum of the powers of $y$ in $w$ is zero.

Let $\pi_{2}$ be the projection $\pi_{2}: G * \rightarrow D$, where

$$
D=\left\langle F, t \mid t^{-1} x^{-n} y x^{n} t=y^{-n} x y^{n}, \quad n \in \mathbb{Z}\right\rangle
$$

and $\pi_{2}(h)=1$ for all $h \in G$. Then, $\pi_{2}(w)$ is a non-trivial element (since $w \in A$ ) and has Linnell's property. It follows that there exists $f \in D$ with $|f|$ even such that $f^{-1} \pi_{2}(w) f=\pi_{2}(w)^{m}$ for some $m \in \mathbb{N}, m>0$. (Indeed, there exist $f_{0} \in D$ and $k \in$ $\mathbb{N}, k>0$, such that $f_{0}^{-1} \pi_{2}(w) f_{0}=\pi_{2}(w)^{k}$. Then, $\left|f_{0}^{2}\right|$ is even and $f_{0}^{-2} \pi_{2}(w) f_{0}^{2}=$ 
$\pi_{2}(w)^{k^{2}}$.) In view of the previous lemma, $\pi_{2}(w)$ is conjugate with $\pi_{2}(w)^{m}$ in $F$. But then we have a non-trivial element of $F$ that is conjugate to one of its powers in $F$, which is impossible (cf. [15, Theorem 4.6]). The contradiction shows that $u$ cannot be conjugate to any element $w \in A$ and hence $C_{G *}(u) \subseteq G * F$.

Furthermore, if $T$ is the tree associated to the group $G *$, then $u$ fixes only one vertex since in the opposite case, it would fix an edge (cf. the proof of Lemma 3.1) and then $u$ would be conjugate to an element of $A$. It follows just as in the proof of Lemma 3.1 that $u$ has Linnell's property in $G * F$.

Remark 4.11. From the proof above it follows that the group $D$ defined in the statement of Lemma 4.9 contains no element having Linnell's property. It follows that $D \in \mathcal{A}_{L}$.

Theorem 4.12. Let $G$ be a countable group and $G *$ the $H N N$-extension defined in the proof of Theorem 4.7. If $G \in \mathcal{A}_{L}$ then $G * \in \mathcal{A}_{L}$.

Proof. Let $g \in G *$ be an element having Linnell's property. Since the cap product map that we are interested in is not surjective if the cap product map associated with any conjugate of $g$ is not, we may invoke Corollary 4.10 and assume that $C_{G *}(g) \subseteq G * F$, with $g$ having Linnell's property in $G * F$. In that case, the extension

$$
1 \longrightarrow \mathbb{Z} \stackrel{g}{\longrightarrow} C_{G *}(g) \longrightarrow N_{G *}(g) \longrightarrow 1
$$

coincides with the extension

$$
1 \longrightarrow \mathbb{Z} \stackrel{g}{\longrightarrow} C_{G * F}(g) \longrightarrow N_{G * F}(g) \longrightarrow 1
$$

Since $G * F \in \mathcal{A}_{L}$, the cap product map (1) is not surjective for $n \gg 0$.

\section{References}

[1] H. Bass, Euler characteristics and characters of discrete groups, Invent. Math. 35 (1976), 155-196.

[2] H. Bass, Traces and Euler characteristics, in Homological group theory (Proc. Sympos., Durham, 1977), London Math. Soc. Lecture Note Ser. 36 (1979), $1-26$.

[3] B. Baumslag, Residually free groups, Proc. London Math. Soc. 17 (1967), 402418.

[4] D. Burghelea, The cyclic homology of the group rings, Comment. Math. Helv. 60 (1985), 354-365.

[5] A. Connes, Non-commutative differential geometry, Publ. Math. IHES 62 (1985), 41-144.

[6] B. Eckmann, Cyclic homology of groups and the Bass conjecture, Comment. Math. Helv. 61 (1986), 193-202.

[7] I. Emmanouil, On a class of groups satisfying Bass' conjecture, Invent. Math. 132 (1998), 307-330.

[8] I. Emmanouil, Nilpotent cohomology classes and some examples of groups, $J$. Pure Applied Algebra 163 (2001), 159-171. 
[9] I. Emmanouil, Idempotent matrices over complex group algebras, SpringerVerlag, New York (2006).

[10] I. Emmanouil and Inder Bir S. Passi, A contribution to Bass' conjecture, J. Group Theory 7 (2004), 409-420.

[11] I. Emmanouil and Inder Bir S. Passi, Group homology and Connes' periodicity operator, J. Pure Applied Algebra 205 (2006), 375-392.

[12] M. Karoubi, Homologie cyclique et K-théorie, Astérisque 149 (1987), Soc. Math. France, Paris, 147 pp.

[13] P.A. Linnell, Decomposition of augmentation ideals and relation modules, Proc. London Math. Soc. 47 (1983), 83-127.

[14] R.C. Lyndon and P.E. Schupp, Combinatorial group theory, Springer-Verlag, New York (1977)

[15] W. Magnus, A. Karrass and D. Solitar, Combinatorial Group Theory: Presentations of groups in terms of generators and relations (second edition), Dover Publ. Inc., New York (1976).

[16] J.A. Moody, Induction Theorems for Infinite Groups, Ph.D. thesis, Columbia University, 1986.

[17] J.J. Rotman, An introduction to the theory of groups (fourth edition), Grad. Texts. in Math. 148, Springer-Verlag, New York (1995).

[18] J.A. Schafer, Traces and the Bass conjecture, Michigan Math. J. 38 (1991), 103-109.

[19] J-P. Serre, Trees, Springer-Verlag, New York, 1980.

[20] A. Strojnowski, On Bass' "strong conjecture" about projective modules, J. Pure Appl. Algebra 62 (1989), 195-198.

[21] M. Sykiotis, Centralizers on groups acting on trees and Bass' conjecture, Comm. Algebra 30 (2002), 1199-1206.

[22] M. Sykiotis, On two classes of groups satisfying the strong Bass conjecture, Comm. Algebra 30 (2002), 4425-4436.

Dimitrios Panagopoulos dipanag@math.uoa.gr

Department of Mathematics, University of Athens, Athens, Greece 